UVX 2012, 01002 (2013)

DOI: $10.1051 /$ uvx/201301002

(C) Owned by the authors, published by EDP Sciences, 2013

\title{
Performances d'une source de rayonnement extrême ultraviolet (EUV) par décharge capillaire à effet cathode creuse
}

\author{
O. Sarroukh ${ }^{1}$, P. Choi ${ }^{1}$, O. Benali ${ }^{1}$, S. Zakharov ${ }^{1,2}$, V. S. Zakharov ${ }^{1}$ \\ et C. Dumitrescu ${ }^{1}$ \\ ${ }^{1}$ EPPRA SAS, 16 Av. du Québec, SILIC 706, 91140 Villebon-sur-Yvette, France \\ ${ }^{2}$ RRC Institut Kurchatov, Moscou, Russie
}

\begin{abstract}
Résumé. Depuis Plusieurs années, les sources de rayonnement Extrême Ultraviolet (EUV) suscitent un intérêt de plus en plus considérable pour de nombreuses applications scientifiques et technologiques. A Eppra, nous développons une source de rayonnement nommée Cyclops émettant dans le domaine de l'extrême ultraviolet EUV et des X mous destinée aux applications liées à la nouvelle génération de lithographie et à la biologie. Le principe de cette source pulsée consiste en une décharge capillaire assistée par effet cathode creuse. Le taux de répétition de la source peut atteindre $5 \mathrm{kHz}$. Nous soulignons que le fonctionnement de la source à haute cadence et à forte énergie injectée engendre une surchauffe de certains composants de la source et limite ainsi sa durée de vie. Dans cet article, il serait question de présenter les principales performances de cette source en particulier les résultats en terme d'éclairement, d'étendue spectrale et de puissance dans une bande passante de $3 \mathrm{~nm}$ autour de $13,4 \mathrm{~nm}$.
\end{abstract}

\section{INTRODUCTION}

L'étape clef dans l'industrie de la microélectronique est la lithographie optique qui permet d'imprimer les motifs des circuits intégrés sur des résines photosensibles. La miniaturisation des circuits intégrés est guidée par une feuille de route internationale, appelée ITRS (de l'anglais International Technology Roadmap for Semiconductor), éditée et revue régulièrement par des experts industriels. Elle permet de donner des spécifications en termes de performances, de coût des systèmes microélectroniques et de dimensions critiques [1]. Afin de pouvoir rester dans la course vers la miniaturisation, la lithographie EUV est proposée depuis quelques années comme solution pour remplir ces exigences industrielles. En réduisant la longueur d'onde d'exposition, la lithographie EUV à $13,5 \mathrm{~nm}$ permet de diminuer la résolution des outils lithographiques actuels et atteindre ainsi des résolutions inférieures à $22 \mathrm{~nm}$. Bien qu'il existe parallèlement d'autres méthodes de fabrication des circuits intégrés à savoir la lithographie par double impression ou la lithographie à faisceau d'électrons appelée également la lithographie électronique, aucune n'a atteint la maturité suffisante pour assurer la cadence d'impression nécessaire à la production en masse. Dans ce contexte, EPPRA développe une source de rayonnement EUV dont le plasma émetteur est généré par décharge capillaire à effet cathode creuse. Il s'agit d'une décharge pulsée et dont l'impulsion de courant présente une amplitude de plusieurs kiloampères et un temps de monté très rapide qui ne dépasse pas une dizaine de nanosecondes. Le plasma créé par ce type de décharge permet d'atteindre des températures électroniques comprises entre 20 et $40 \mathrm{eV}$ et des densités électroniques de l'ordre de $2 \mathrm{E} 17 \mathrm{~cm}^{-3}$. Ces paramètres on été obtenus par simulation grâce à un code de calcul magnétohydrodynamique [2]. De plus la géométrie de la cathode ainsi que la pression du mélange gazeux favorise la génération d'un faisceau d'électrons dans l'axe du capillaire et sa propagation permet

This is an Open Access article distributed under the terms of the Creative Commons Attribution License 2.0, which permits unrestricted use, distribution, and reproduction in any medium, provided the original work is properly cited. 


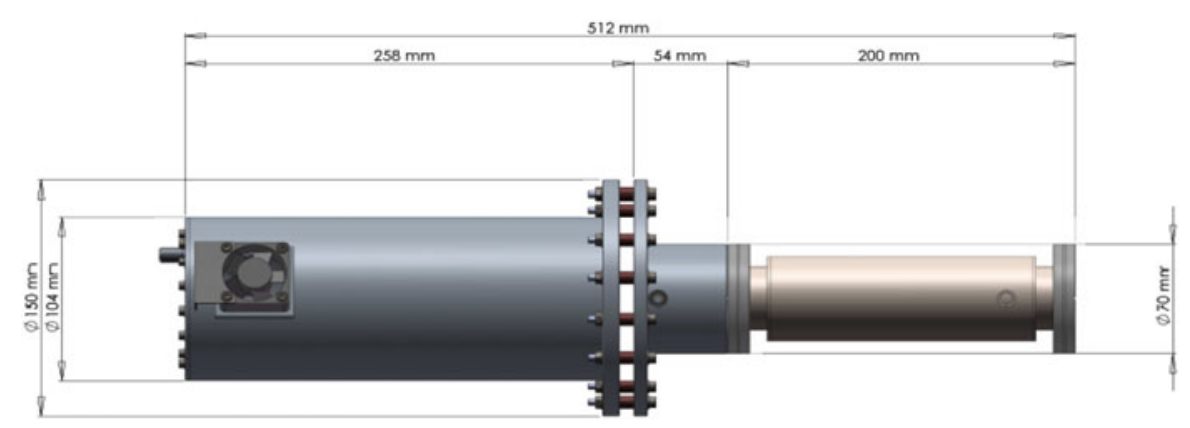

Figure 1. Schéma de la source Cyclops.

l'ionisation initiale du gaz et un meilleur transfert d'énergie aux espèces circulant dans le gaz. Cyclops présente un spectre d'émission assez large qui s'étend du rayonnement visible au rayonnement X mous. Nous avons étudié l'influence de plusieurs paramètres de la décharge afin de déterminer les conditions optimales pour un meilleur rendement dans le domaine spectrale de l'extrême ultraviolet plus particulièrement autour de $13,5 \mathrm{~nm}$.

Le travail mené ici a pour but de déterminer les performances du faisceau EUV de Cyclops. Nous montrerons que l'originalité de cette source réside dans son faible degré de divergence grâce à une structure plasma active située à une dizaine de centimètres du capillaire permettant une fonction de focalisation pour collecter et projeter le rayonnement EUV.

\section{DISPOSITIF EXPÉRIMENTAL}

Le dispositif expérimental de Cyclops regroupe essentiellement deux éléments : le cabinet d'instrumentation et la source plasma. Le cabinet regroupe le système de distribution de gaz et du circuit de refroidissement, l'alimentation haute tension et le panneau de control. Le réacteur plasma est présenté en figure 1, il pèse $7 \mathrm{~kg}$ et composé de trois parties à savoir la cathode, l'anode et structure de collection. Cette dernière constitue la terminaison de système à travers laquelle se connecte à d'autres dispositifs d'illumination ou bancs d'expérience.

L'alimentation haute tension permet de stocker l'énergie électrique dans un banc de condensateurs ayant une capacité totale de 1,9 nF. L'énergie ainsi stockée est injectée dans un mélange gazeux circulant dans un capillaire en alumine $\mathrm{Al}_{2} \mathrm{O}_{3}$. Ce mélange gazeux est composé d'un gaz porteur léger, généralement l'hélium et d'autres gaz émetteurs. Le choix de ces derniers dépend de la gamme spectrale dans laquelle la source doit émettre. Nous avons trouvé que le rayonnement EUV est plus intense lors de l'utilisation d'un mélange composé de $\mathrm{He} / \mathrm{N}_{2} / \mathrm{Xe}$. Ce mélange est injecté continuellement dans un capillaire et sa pression est maintenue à quelques dizaines de millitorr en sortie du capillaire. La tension de charge appliquée aux bornes des électrodes situées aux extrémités du capillaire peut atteindre $26 \mathrm{kV}$. L'énergie ainsi stockée par pulse est comprise entre 200 et $500 \mathrm{~mJ}$. La source opère en mode pulsé et la fréquence de tir est comprise entre 1 et $5 \mathrm{kHz}$. La partie cathode de la source est refroidie par un ventilateur et l'anode est refroidie grâce une circulation d'eau ayant un débit de 31/min qui la maintient à une température d'environ $19^{\circ}$.

\section{MESURES OPTIQUES}

La caractérisation temporelle du rayonnement EUV se fait moyennant une photodiode filtrée SXUV 20 A MoSi de chez IRD permettant de détecter les photons de longueurs d'onde comprise entre 12,2 et $15,8 \mathrm{~nm}$. Afin de s'affranchir du rayonnement visible et infra rouge, nous couplons également cette 


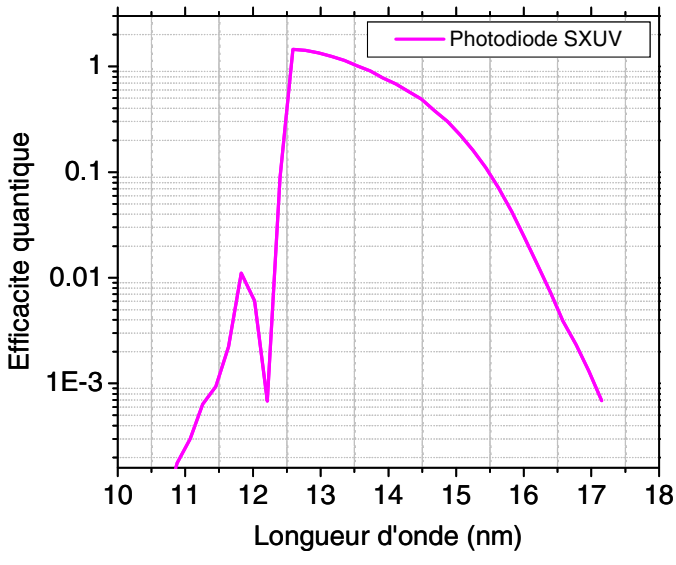

a)

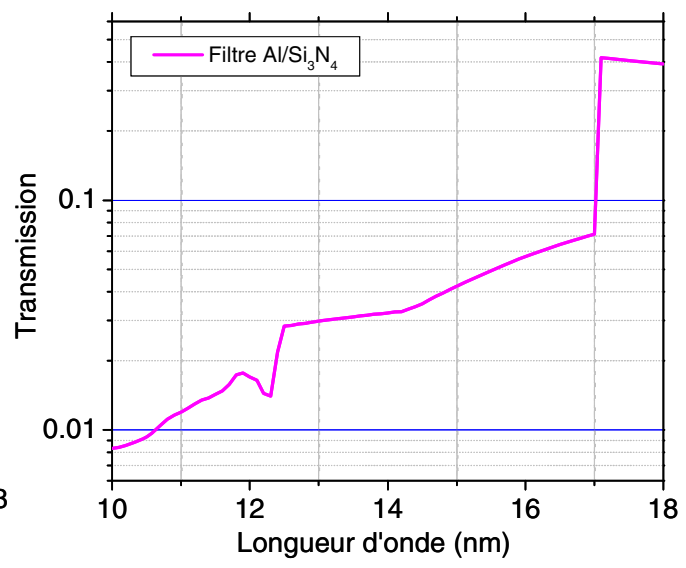

b)

Figure 2. Courbe d'efficacité quantique de la photodiode filtrée en fonction de la longueur d'onde des photons (a) et courbe de transmission du filtre utilisé (b).

photodiode à un filtre composé d'une couche de nitrure de silicium $\mathrm{Si}_{3} \mathrm{~N}_{4}$ de $50 \mathrm{~nm}$ d'épaisseur et ayant d'une part et d'autre une couche de $55 \mathrm{~nm}$ d'épaisseur d'aluminium. Nous superposons à ce filtre un diaphragme de $200 \mu \mathrm{m}$ de diamètre qui définit une surface active de détection de $0,031 \mathrm{~mm}^{2}$. La courbe d'efficacité de la photodiode ainsi que la transmission totale du filtre sont représentés en figure 2. La première est donnée par le constructeur et la deuxième courbe a été calculée [3]. La plage spectrale de détection définie par ce filtre et la photodiode est comprise entre 12,4 et 15,4 nm. Nous remarquons que la transmission totale du filtre à 13,5 nm à tenir en compte lors du calcul du flux lumineux est de $3 \%$.

L'ensemble composé de la photodiode, filtre et diaphragme est disposé sur un bras amovible permettant d'exercer un mouvement de translation perpendiculaire à l'axe optique avec un pas de $500 \mu \mathrm{m}$. Il est donc possible de détecter à chaque position l'impulsion temporelle des photons EUV émis. Notons que le flux d'énergie lumineuse est proportionnel à l'intégrale du signal délivré par la photodiode.

Nous avons placé le système de détection décrit dans le paragraphe précédent à une distance de $62 \mathrm{~cm}$ du capillaire et nous avons enregistré l'impulsion temporelle du rayonnement émis de 12,4 à $15,4 \mathrm{~nm}$ de l'axe vertical perpendiculaire à l'axe optique. Nous rappelons que le flux lumineux est proportionnel à l'intégrale du signal de la photodiode. Lors de ces mesures la source opérait à un taux de répétition de $1 \mathrm{kHz}$ et l'énergie injectée dans le mélange gazeux composé de $\mathrm{He} / \mathrm{N}_{2} / \mathrm{Xe}$ est de $450 \mathrm{~mJ}$. Nous traçons ensuite l'évolution de l'amplitude maximale de cette impulsion en fonction de la position verticale. Les résultats présentés en figure 3, peuvent être ajustés à un profil Gaussien. Nous sommes donc capable de définir la taille du faisceau à partir du profil ajusté. En effet le rayon correspond à la demi-largeur à $1 / \mathrm{e}^{2}$ de la gaussienne ajustée. Notons que l'éclairement maximal le long de l'axe du capillaire est de $4,210^{17} \mathrm{ph} / \mathrm{cm}^{2} / \mathrm{s}$ et que la puissance rayonnée est de $4,8 \mathrm{~W}$. Bien entendu, le calcul assume une symétrie radiale et prend en considération que le profil est parfaitement gaussien.

La puissance et l'éclairement sont deux paramètres qui dépendent fortement de l'énergie injectée, de la pression et de la composition du mélange gazeux. L'optimisation du mélange gazeux en terme de pression et de débit a permis d'atteindre une puissance et un éclairement à un taux de répétition de $3 \mathrm{kHz}$ de $16 \mathrm{~W}$ et $2,5 \mathrm{E} 17 \mathrm{ph} / \mathrm{cm}^{2} / \mathrm{s}$ respectivement.

En figure 4, le pic d'éclairement des photons émis dans la bande de $3 \mathrm{~nm}$ autour de 13,4 nm est présenté en fonction de l'énergie injectée à un taux de répétition de $1 \mathrm{kHz}$ et à une distance de $62 \mathrm{~cm}$ du capillaire. Sous réserve de mesures à des énergies injectées qui ne peuvent pas être atteintes ici, 


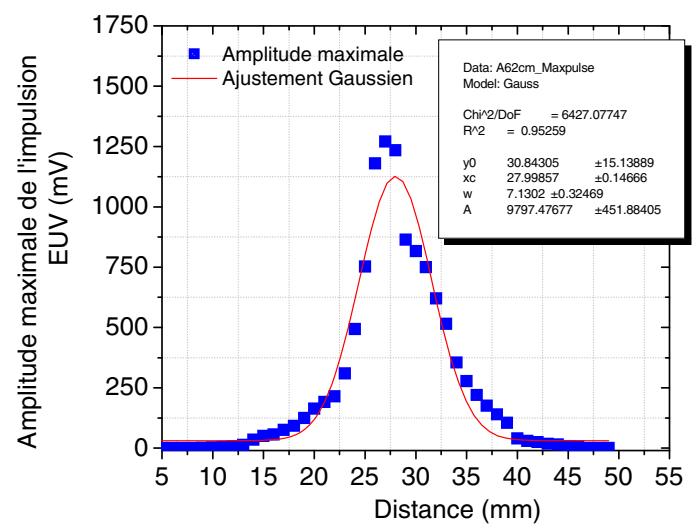

Figure 3. Profils mesuré et ajusté du faisceau EUV à $62 \mathrm{~cm}$ de la source pour une tension de $21 \mathrm{kV}$ et une capacité totale de $1,9 \mathrm{nF}$.

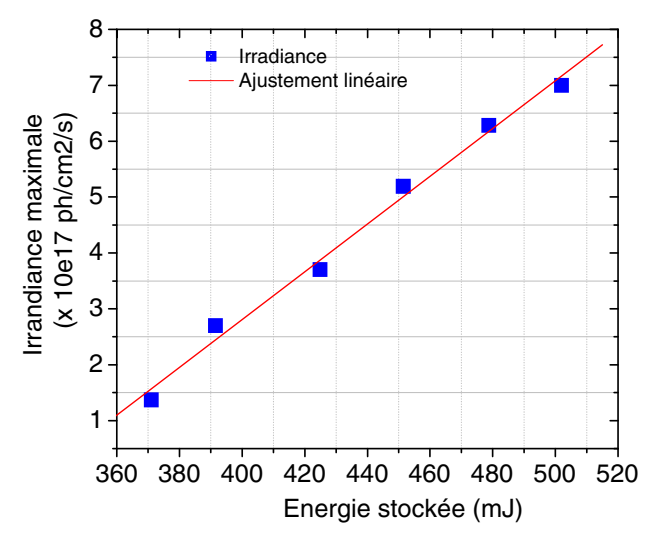

Figure 4. Évolution de l'éclairement des photons EUV émis en fonction de l'énergie injectée.

l'éclairement des photons est d'autant plus importante que l'énergie injectée est élevée. Notons que la température électronique augmente quand l'énergie injectée dans la plasma est plus élevée et par conséquent l'émission du plasma autour de $13,5 \mathrm{~nm}$ due essentiellement aux ions de xénon $\mathrm{Xe}^{10+}$ domine le spectre. Nous avons donc tout intérêt que la source soit capable d'assurer un fonctionnement à des énergies injectées plus élevées.

Même si le xénon est un excellent émetteur dans la région spectrale de l'EUV, il présente certaines limitations physiques. L'étain présente un coefficient de conversion EUV plus important que le xénon [4] mais malheureusement son utilisation à forte concentration induit des débris dans le système. Ces derniers polluent les optiques utilisées et modifient leurs propriétés. En effet, l'introduction de l'étain dans le mélange gazeux induit une augmentation de rendement du rayonnement EUV. Nous avons trouvé que l'utilisation des électrodes à base d'un alliage contenant l'étain provoque une augmentation de presque $40 \%$ de nombre de photons EUV. Nous avons pu mesurer un éclairement de $1,2 \mathrm{E} 18 \mathrm{ph} / \mathrm{cm}^{2} / \mathrm{s}$ à $1 \mathrm{kHz}$ et pour une énergie injectée de $440 \mathrm{~mJ}$. Le rendement EUV est d'autant plus élevée que la concentration d'étain dans l'alliage composant la cathode est forte ce pendant la source devient moins stable au-delà d'une certaine concentration critique. 
Tableau 1. Divergence et angle solide du rayonnement émis dans la bande spectrale de 12,4 à 15,4 nm.

\begin{tabular}{|c|c|c|c|}
\hline $\begin{array}{c}\text { Distance entre } \\
\text { la source et le } \\
\text { capillaire } \mathbf{( c m )}\end{array}$ & $\begin{array}{c}\text { Éclairement à } \mathbf{1 ~ k H z} \\
\left(\mathbf{P h} / \mathbf{c m}^{\mathbf{2}} \mathbf{/ S}\right)\end{array}$ & $\begin{array}{c}\text { Demi largeur à mi } \\
\text { hauteur HWHM } \\
(\mathbf{m m})\end{array}$ & $\begin{array}{c}\text { Demi angle de } \\
\text { divergence } \theta \\
(\mathbf{d e g})\end{array}$ \\
\hline 74 & $8,2 \mathrm{E} 17$ & 6,13 & \multirow{2}{*}{0,8} \\
\hline 98 & $1,8 \mathrm{E} 17$ & 9,65 & \\
\hline \multicolumn{2}{|c|}{ Angle solide de rayonnement $=2 * \pi(1-\cos \theta)=6 \mathrm{E}-4 \mathrm{sr}$} \\
\hline
\end{tabular}

\section{COMPORTEMENT SPATIAL DU RAYONNEMENT EUV}

Nous suivons dans ce paragraphe l'évolution de la taille du faisceau EUV à deux distances différentes du capillaire. Il est donc possible de déterminer à la fois l'angle solide d'émission et l'étendue spectrale du rayonnement. Le tableau 1 regroupe les résultats obtenus pour un mélange $\mathrm{He} / \mathrm{Ar} / \mathrm{Xe}$. Nous trouvons que la taille du faisceau s'élargit d'un facteur d'environ 1.6 sur une distance de $24 \mathrm{~cm}$ ce qui correspond à un demi-angle de divergence de $0,8^{\circ}$. L'angle solide d'émission est de $6 \mathrm{E}-4 \mathrm{sr}$ et l'étendue spectrale est de $1,7 \mathrm{E}-2 \mathrm{~mm}^{2}$.sr. Nous soulignons que ces paramètres sont plus faibles lors de l'utilisation d'un mélange $\mathrm{He} / \mathrm{N}_{2} / \mathrm{Xe}$. En effet, le demi-angle de divergence se trouve diminué d'un facteur 2. Les propriétés de collimation de la structure plasma se trouvant en sortie de la source sont plus efficaces lors de l'utilisation de l'argon dans le mélange gazeux.

Grâce à ces propriétés optiques de la source et ses faibles dimensions physiques, nous avons pu montrer la faisabilité du multiplexage de plusieurs sources. Nous pouvons faire opérer jusqu'à huit sources de façon simultanée ou alternée et les faire converger en un seul point. Ceci constitue une façon moins complexe d'augmenter davantage les performances de cette source et dont les contraintes sont plutôt d'ordre mécaniques et électroniques.

\section{CONCLUSION}

La source Cyclops est désormais opérationnelle et opère de façon satisfaisante dans la gamme spectrale comprise entre 12,4 et $15,4 \mathrm{~nm}$. Nous avons maîtrisé les problèmes thermiques rencontrés lors de fonctionnement de la source à haute fréquence et à forte densité d'énergie injectée. En effet, il est possible de travailler dans un régime où l'énergie stockée est de $500 \mathrm{~mJ}$ à un taux de répétition de $3 \mathrm{kHz}$. Au-delà de ce régime la durée de vie de certains composants à savoir les électrodes et le collecteur se trouve fortement diminuée. Le but des prochaines expériences est donc de s'affranchir de ces problèmes et d'améliorer ainsi la durée de vie de la source tout en continuant d'augmenter les performances.

\section{Remerciements}

Ce travail a bénéficié du soutien de OSEO et également du gouvernement d'investissement de RAK.

\section{Références}

[1] Iternational Technology Roadmap for semiconductors, http://www.itrs.net/

[2] http://www.cxro.lbl.gov/

[3] S.V. Zakharov, P. Choi, V.S. Zakharov, Proceeding of SPIE (Editeur : Bruno M. La fontaine) 7636 3A (2010)

[4] J. Pankert, K. Bergmann, J. Klein, W. Neff, O. Rosier, S. Seiwert, C. Smith, S. Probst, D. Vaudrevange, G. Siemons, R. Apetz, J. Jonkers, M. Loeken, E. Bosh, G. Derra, T. Krücken. P. Zink. Proceeding of SPIE, vol 5037 (2003) 